\title{
Methionine Sulfoxides on PrPsc: A Prion-Specific Covalent Signature ${ }^{\dagger}$
}

\author{
Tamar Canello, ${ }^{*}$ Roni Engelstein, ${ }^{\ddagger}$ Ofra Moshel, ${ }^{\S}$ Konstantinos Xanthopoulos, ${ }^{\prime \prime}$ María E. Juanes, ${ }^{\perp}$ Jan Langeveld, \\ Theodoros Sklaviadis," Maria Gasset, ${ }^{\perp}$ and Ruth Gabizon*,* \\ Department of Neurology, The Agnes Ginges Center for Human Neurogenetics, Hadassah University Hospital, \\ 91120 Jerusalem, Israel, Interdepartmental Equipment Unit, The Hebrew University-Hadassah Medical School, \\ 91120 Jerusalem, Israel, School of Pharmacy, Aristotle University of Thessaloniki, 54124 Thessaloniki, Greece, Instituto \\ Química-Física "Rocasolano", CSIC, 28006 Madrid, Spain, and Central Veterinary Institute of Wageningen UR, \\ 8200 AB Lelystad, The Netherlands
}

Received May 3, 2008; Revised Manuscript Received June 19, 2008

\begin{abstract}
Prion diseases are fatal neurodegenerative disorders believed to be transmitted by $\operatorname{PrP}^{\mathrm{Sc}}$, an aberrant form of the membrane protein $\mathrm{PrP}^{\mathrm{C}}$. In the absence of an established form-specific covalent difference, the infectious properties of $\mathrm{PrP}^{\mathrm{Sc}}$ were uniquely ascribed to the self-perpetuation properties of its aberrant fold. Previous sequencing of the $\operatorname{PrP}$ chain isolated from $\operatorname{PrP}(27-30)$ showed the oxidation of some methionine residues; however, at that time, these findings were ascribed to experimental limitations. Using the unique recognition properties of $\alpha \operatorname{PrP} \mathrm{mAb}$ IPC2, protein chemistry, and state of the art mass spectrometry, we now show that while a large fraction of the methionine residues in brain $\mathrm{PrP}^{\mathrm{Sc}}$ are present as methionine sulfoxides this modification could not be found on brain $\operatorname{PrP}^{\mathrm{C}}$ as well as on its recombinant models. In particular, the pattern of oxidation of M213 with respect to the glycosylation at $\mathrm{N} 181$ of $\mathrm{PrP}^{\mathrm{Sc}}$ differs both within and between species, adding another diversity factor to the structure of $\mathrm{PrP}^{\mathrm{Sc}}$ molecules. Our results pave the way for the production of prion-specific reagents in the form of antibodies against oxidized PrP chains which can serve in the development of both diagnostic and therapeutic strategies. In addition, we hypothesize that the accumulation of $\mathrm{PrP}^{\mathrm{Sc}}$ and thereafter the pathogenesis of prion disease may result from the poor degradation of oxidized aberrantly folded PrP.
\end{abstract}

Many lines of evidence point to $\mathrm{PrP}^{\mathrm{Sc}}$ being the only active constituent of the infectious prion, the agent that causes fatal neurodegenerative prion diseases such as scrapie, bovine spongiform encephalopathy (BSE), and Creutzfeldt-Jakob disease (CJD) (1). In the absence of convincing data to the contrary, $\mathrm{PrP}^{\mathrm{Sc}}$ and its normal isoform, $\mathrm{PrP}^{\mathrm{C}}$, are believed to differ from each other only in their high-order structures, mostly an $\alpha$-helical fold for $\operatorname{PrP}^{\mathrm{C}}$ and largely a $\beta$-sheet assembly for $\operatorname{PrP}^{\mathrm{Sc}}(2)$. The established covalent modifications on both prion protein chains include two N-linked glycosylation sites (residues 181 and 197 in SHaPrP) and a glycosyl-phosphatidyl-inositol (GPI) moiety, which anchors the protein molecules to membrane rafts (3). Although some differences at the sugar moieties were suggested, no "prionspecific feature" was identified at this level since both the conversion of $\mathrm{PrP}^{\mathrm{C}}$ to $\mathrm{PrP}^{\mathrm{Sc}}$ and the propagation of prion infectivity also occur in the absence of $\mathrm{N}$-linked glycosylation and of the GPI anchor (4-6). Furthermore, cysteines 179 and

\footnotetext{
$\dagger$ This work was supported by EU Grant FOOD-CT-2004-506579 (R.G., M.G., J.L., and T.S.), MEC Grant SAF2006-0418 (M.G.), and the Horowitz Foundation (R.G.). The authors have no financial interest in this project.

* To whom correspondence should be addressed: Department of Neurology, The Agnes Ginges Center for Human Neurogenetics, Hadassah University Hospital, Jerusalem. Israel. E-mail: gabizonr@ hadassah.org.il. Telephone: +972-2-6777858. Fax: +972-2-6429441.

$¥$ Hadassah University Hospital.

$\S$ The Hebrew University-Hadassah Medical School.

"Aristotle University of Thessaloniki.

${ }^{\perp}$ CSIC.

\# Central Veterinary Institute of Wageningen UR.
}

214 are engaged in the single intramolecular disulfide bond 42 which links two of the three $\alpha$-helices of $\operatorname{PrP}^{\mathrm{C}}(7)$. An intact, 43 oxidized, disulfide bond was suggested to be essential not 44 only for the conversion of $\mathrm{PrP}^{\mathrm{C}}$ into $\mathrm{PrP}^{\mathrm{Sc}}$ but also for the propagation of prion infectivity $(8)$.

Surprisingly, the biochemical search for differential covalent modifications on $\mathrm{PrPS}^{\mathrm{Sc}}$ is summarized in only one report. In this seminal work, Stahl et al. sequenced $\operatorname{PrP}(27-30)$, the purified proteinase $\mathrm{K}(\mathrm{PK})$ resistant form of $\mathrm{SHaPrP}^{\mathrm{Sc}}$ (9). Using mass spectrometry and Edman degradation, they proved that the primary structure of the infectious core of $\mathrm{PrP}^{\mathrm{Sc}}$ is indeed identical to the one deduced from the $\operatorname{PrP}$ gene sequence and subsequently to that of $\operatorname{PrP}^{\mathrm{C}}$. They also found that either one or two methionines (M206 and/or M213) were present in the form of methionine sulfoxide (MetO), ${ }^{1}$ but in the absence of controls such as recombinant models (recPrP) or purified $\operatorname{PrP}^{\mathrm{C}}$, the relevance of this oxidation could not be assured at that time (9).

Stress-related oxidation of amino acids, in particular methionines, is more prominent at older ages (10). Concomitantly, the activity of enzymes which recycle oxidized proteins by reducing MetO, methionine reductases, is compromised at older ages (11). Reactive oxygen species (ROS) formed during oxidative stress events play a role in

\footnotetext{
${ }^{1}$ Abbreviations: MetO, methionine oxide; MMA, $N$-methylmercaptoacetamide; PK, proteinase K; ROS, reactive oxygen species; rMoPrP, recombinantly produced mouse $\operatorname{PrP}(23-232)$ chain; $r S H a P r P$, recombinantly produced Syrian hamster $\operatorname{PrP}(23-232)$ chain.
} 
the pathology of neurodegenerative disorders such as Alzheimer's and Parkinson's diseases (12), probably since the oxidation of proteins by these species alters their hydrophilicity and enhances their protease resistance (13). Consistent with its unique large number of methionines, $\operatorname{PrP}^{\mathrm{C}}$ has been assigned a role in the protection of cells from $\operatorname{ROS}(14,15)$. In this sense, several experimental setups indicated that recPrP constitutes an excellent target for oxidation $(16,17)$. However, the cross talk between PrP oxidation and the conversion to its $\beta$-form is unclear. While some experiments indicate that, as for other proteins (13), oxidation of $\alpha$-helixfolded recPrP (modeling for $\mathrm{PrP}^{\mathrm{C}}$ ) favors the formation and aggregation of an enriched $\beta$-sheet conformer structure (reminiscent of $\mathrm{PrP}^{\mathrm{Sc}}$ ), other experiments show convincingly that the oxidization of $\alpha$-recPrP drastically decreases the rate and efficiency of the conversion into amyloid-like $\beta$-recPrP polymers (18). The last possibility would infer that oxidation of methionines in $\mathrm{PrP}^{\mathrm{Sc}}$, as suggested by Stalh et al., occurs after the conversion of $\mathrm{PrP}^{\mathrm{C}}$ to the $\beta$-isoform. Alternatively, this would also support the idea that the $\operatorname{PrP}^{\mathrm{Sc}}$ chains carrying the oxidized methionines are the true infectious entities.

Using both the reactivity of IPC2, an $\alpha$-PrP mAb recognizing the intramolecular disulfide bond and its proximal environment in $\mathrm{PrP}^{\mathrm{C}}$ but not in $\mathrm{PrP}^{\mathrm{Sc}}$, and mass spectrometry, we show here, for the first time, that methionine residues in $\mathrm{PrP}^{\mathrm{Sc}}$ are indeed present as methionine sulfoxides. This is not the case for $\mathrm{PrP}^{\mathrm{C}}$ or for its recombinant models (recPrP). Therefore, methionine oxidation constitutes a specific covalent modification of PrP in its prion state. Whether oxidation of methionines in the $\operatorname{PrP}$ chains is a requirement for the induction of the proper infectious folding remains to be established.

\section{MATERIALS AND METHODS}

Production of Full-Length recPrP. Full-length chains corresponding to the mouse, hamster, human, ovine, and bovine PrP sequences lacking the $\mathrm{N}$ - and C-terminal sequences were expressed from their pET11a construct and folded to their $\alpha$-form as described previously (21). Their $\beta$-forms were obtained after prolonged incubation in $30 \mathrm{mM}$ $\mathrm{NaAc}(\mathrm{pH} 5.0)$ containing $0.2 \mathrm{M} \mathrm{NaCl}$ and $3 \mathrm{M}$ urea at $37{ }^{\circ} \mathrm{C}(19,20)$. The rSHaPrP(23-232) N181D and M213S mutants were generated by site-directed mutagenesis using QuickChange protocols and the following oligos (only forward sequences are given): 5'-CGATTGTGTCGACATCACCATCAAGCAGC (N181D) and 5'-GTGGTGGAGCAGTCCTGTACCACCCAG (M213S).

Normal and Prion-Infected Brain Homogenates. Brains from normal and Sc237 prion-infected Syrian hamsters and of normal and RML-infected C57 black mice were obtained from the Hadassah Animal Facility. Human brain samples from control, sporadic CJD cases and from E200K genetic cases were supplied from the Surveillance Service of Hadassah Hospital. Control and scrapie-affected ovine brain samples were provided by the Agriculture Center of the Israeli Ministry of Agriculture. Normal and prion-containing brain samples were homogenated at $10 \%(\mathrm{w} / \mathrm{v})$ in $10 \mathrm{mM}$ Tris ( $\mathrm{pH}$ 7.4) and 0.3 M sucrose. Unless stated, proteinase $\mathrm{K}$ (PK) digestions were performed by incubating $50 \mu \mathrm{L}$ of $10 \%$ prion-infected brain homogenates in $40 \mu \mathrm{g} / \mathrm{mL}$ protease for $1 \mathrm{~h}$ at $37^{\circ} \mathrm{C}$. For the $\operatorname{SHaPrP}(27-30)$ enrichment, brain microsomes from infected animals were extracted in TNS buffer (10 mM Tris, $100 \mathrm{mM} \mathrm{NaCl}$, and 2\% Sarkosyl), cooled for $1 \mathrm{~h}$ at $4{ }^{\circ} \mathrm{C}$, and then ultracentrifuged for $1 \mathrm{~h}$ at $100000 \mathrm{~g}$. Pellets were resuspended in $100 \mu \mathrm{L}$ of TNS and digested for $1 \mathrm{~h}$ with $100 \mu \mathrm{g} / \mathrm{mL}$ proteinase $\mathrm{K}$. After the addition of $1 \mathrm{~mL}$ of TNS, samples were centrifuged again for $1 \mathrm{~h}$ at $40000 \mathrm{rpm}$.

Antibody Generation. Full-length $\alpha$-rMoPrP prepared as described above in combination with pertussis toxin and Friend's adjuvant was used to immunize PrP-ablated mice (21). Subsequently, spleens from the immunized mice were processed for the preparation of mAbs. Hybridomas were screened by an ELISA against rMoPrP and then tested for immunoblotting. PEPscan analysis was performed for the identification of $\alpha$-PrP mAb epitopes (22).

$\mathrm{H}_{2} \mathrm{O}_{2}$ Oxidation. Oxidation of $\mathrm{rPrP}$ with $\mathrm{H}_{2} \mathrm{O}_{2}$ was performed essentially as described previously (17). Briefly, recPrP (approximately $2 \mu \mathrm{M}$ in PBS) was incubated in the absence or presence of 3,10 , and $50 \mathrm{mM} \mathrm{H}_{2} \mathrm{O}_{2}$. After incubation for $15 \mathrm{~min}$ at $37{ }^{\circ} \mathrm{C}$, the reaction was quenched by addition of $20 \mathrm{mM}$ free methionine.

$N$-Methylmercaptoacetamide Reduction. Normal and proteinase K-digested prion-infected brain homogenates were treated with $6 \mathrm{M} \mathrm{N}$-methylmercaptoacetamide (MMA) (23). After incubation for $15 \mathrm{~h}$ at $37^{\circ} \mathrm{C}$, samples were precipitated with 9 volumes of methanol $\left(1 \mathrm{~h},-80{ }^{\circ} \mathrm{C}\right)$ and then centrifuged (10000 rpm, $30 \mathrm{~min}, 4{ }^{\circ} \mathrm{C}$ ). For $\mathrm{H}_{2} \mathrm{O}_{2}$-treated and untreated recPrP samples, MMA was added at a concentration of $2 \mathrm{M}$ in PBS. After incubation for $2 \mathrm{~h}$ at $37{ }^{\circ} \mathrm{C}$, proteins were mixed with $40 \mu \mathrm{g}$ of BSA and precipitated with 9 volumes of methanol. For both types of samples, pellets were washed twice with methanol and processed for SDS-PAGE analysis in the absence of $\beta$-mercaptoethanol.

In Situ PNGase Digestion. Enzymatic deglycosylation was adapted from ref 24 . Briefly, after electrotransference, the nitrocellulose membranes were blocked in PBS containing $3 \% \mathrm{BSA}$ for $30 \mathrm{~min}$ and then incubated for $3 \mathrm{~h}$ with $50 \mathrm{mM}$ sodium phosphate $(\mathrm{pH}$ 7.2) containing PNGase $\mathrm{F}$ at a concentration of either 20 units/mL (Sigma) or 500 units/ $\mathrm{mL}$ (New England Biolabs). After being washed, the membranes were blocked in milk and immunoblotted with the appropriate anti-PrP mAbs.

Mass Spectrometry. Gel bands from relevant PrP chains were processed for tryptic digestion as described previously (25). Subsequently, the peptide mixture was solid phase extracted with a $\mathrm{C} 18$ resin-filled tip (ZipTip, Millipore, Billerica, MA) and nanosprayed into the Orbi-trap MS system in a $50 \% \mathrm{CH}_{3} \mathrm{CN} / 1 \% \mathrm{CHOOH}$ solution. Mass spectrometry was carried out with Orbi-trap (Thermo Finnigen) using a nanospray attachment (26). Data analysis was carried out using Bioworks version 3.3, and database searches were performed with the sequest package and the Mascot package (Matrix Science).

SDS-PAGE, Western Blotting, and ELISA. Proteinase $\mathrm{K}$-treated or untreated brain homogenates as well as recombinant PrP protein samples were denatured in the presence or absence of $\beta$ me and then subjected to SDS-PAGE using either 12\% (brain homogenates) or 14\% (recombinant PrP chains) acrylamide gels. For immunoblotting, the gels were transferred to nitrocellulose membranes and developed with alkaline phosphatase-labeled anti-mouse reagents. For ELISA 


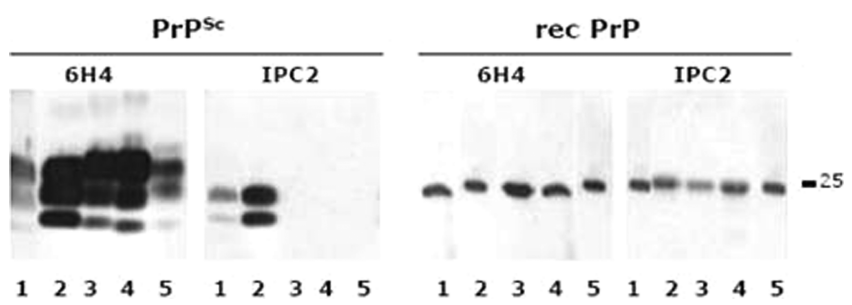

FIGURE 1: PrP recognition by IPC2 required an intact disulfide bond. Differential immunoreactivity of IPC2 and 6H4 anti-PrP mAbs against (1) hamster, (2) mouse, (3) bovine, (4) ovine, and (5) human PrP from proteinase K-digested $10 \%$ (w/v) brain homogenates (10 $\mu \mathrm{L}$ ) and recombinant chains (30 ng). Samples were denatured under reducing conditions before SDS-PAGE analysis.

tests, recombinant PrP and MOG (myelin oligodendrocyte glycoprotein) proteins $(5 \mu \mathrm{g} / \mathrm{mL})$ were bound to the wells of a 96-well plate and reacted with a 1:3000 dilution of IPC1, IPC2, and a control mAb glycoprotein. Measurements were conducted in triplicate, and displayed data correspond to the average of two independent experiments.

\section{RESULTS}

Recognition of PrP by IPC2 Requires an Oxidized Disulfide Bond. Our interest in the search for a prion-specific covalent modification resulted from an investigation into the peculiar properties of IPC2, an anti-PrP mAb prepared in our laboratory against $\alpha-\operatorname{MoPrP}(23-230)$. As depicted in Figure 1, both IPC2 and $6 \mathrm{H} 4 \mathrm{mAb}$ easily recognized rPrPs from an array of species (27). However, when probed with brain homogenates, as opposed to $6 \mathrm{H} 4$, IPC2 reacted only with the non- and monoglycosylated chains of $\mathrm{MoPrP}^{\mathrm{Sc}}$ and, to a lesser extent, of $\mathrm{SHaPrP}^{\mathrm{Sc}}$. These results suggest that the IPC2 epitope comprises a highly conserved part of the PrP sequence, as shown by its activity against all recPrPs, but that a modification present on native $\mathrm{PrPS}^{\mathrm{Sc}}$ precludes the recognition of some of its denatured chains by this antibody.

PEPscan analysis (24) did not identify any linear PrP sequence as the IPC2 epitope, whereas the test easily identified residues $144-151$ of the $\operatorname{PrP}$ sequence as the epitope for the anti-PrP mAb IPC1 (Figure 2A). We next tested whether the IPC2 epitope could comprise the disulfide bond between $\mathrm{C} 179$ and $\mathrm{C} 214$. Figure 2B shows that while the detection of the PrP polypeptide by IPC1 was unaffected by the presence or absence of $\beta$-mercaptoethanol, the detection of recPrP by IPC2 was significantly improved in the absence of the reducing agent, and it was mostly abolished by iodoacetamide blockage of the reduced cysteines. These results indicate that an intact disulfide bond is required for the recognition of PrP forms by IPC2.

Figure 2C depicts the differential reactivity of IPC2 in comparison with those of other anti-PrP antibodies with normal and prion-infected brain samples immunoblotted in the absence of reducing agents. As for $\mathrm{PrP}^{\mathrm{Sc}}$, IPC2 recognized the non- and monoglycosylated bands of rodent $\mathrm{PrP}^{\mathrm{C}}$, albeit with an affinity lower than those of the other anti-PrP antibodies tested. Most intriguingly, IPC2 also recognized, with low affinity, PrP forms of human and ovine normal brain homogenates, while no protease resistant PrP of these species was revealed by this antibody. This further suggests that a prion-specific covalent modification may hinder the recognition of $\mathrm{PrP}^{\mathrm{Sc}}$ forms by IPC2.
Oxidation of Methionine Residues Impairs the Recognition of $\operatorname{PrP}$ by IPC2. To gain insight into the nature of the modification preventing IPC2 recognition, we first looked into the amino acid residues around the PrP disulfide bond (Figure 2B). Sequence differences at residues $183(\mathrm{~V} / \mathrm{I})$ and 215 (T/I/V) between species were first observed, but since IPC2 recognizes all recPrP chains tested (Figure 1A), these residues are unlikely to carry the chemical modification impeding the recognition.

Almost adjacent to $\mathrm{C} 179$ is $\mathrm{N} 181$, which can provide steric repulsion if it is glycosylated or an N-to-D deamidation if it is enzymatically deglycosylated. On the other side of the disulfide bond (C214) we found M213, which was suggested to be present in $\mathrm{PrP}^{\mathrm{Sc}}$ in an oxidized form (9). To investigate whether modifications in one of these sites may affect the recognition of $\mathrm{PrP}^{\mathrm{Sc}}$ by IPC2, we prepared the rSHaPrP(23-232) N181D and $\operatorname{rSHaPrP}(23-232)$ M213S mutants. Figure $3 \mathrm{~B}$ shows both by an immunoblot and by an ELISA that while IPC2 readily recognized the $\operatorname{rSHaPrP}(23-232)$ N181D peptide, the M213S substitution abolished the recognition of PrP by IPC2. These results indicate that while the IPC2 epitope comprises M213, N181 is not essential for the recognition of PrP by this mAb. Nevertheless, high levels of glycosylation on this site may still interfere sterically with the reactivity of the IPC2 antibody (see below).

We next tested whether modification by oxidation of M213 may hinder the recognition of PrP by IPC2, which was the case for the substitution of this amino acid residue with Ser. For this effect, we oxidized both full-length $\mathrm{rMoPrP}(23-230)$ and $\mathrm{rSHaPrP}(23-232)$, folded in either the $\alpha$ - or $\beta$-form (28), with increasing concentrations of $\mathrm{H}_{2} \mathrm{O}_{2}$ as described previously (17). As a control for the reversibility of oxidation, the samples oxidized with $50 \mathrm{mM} \mathrm{H}_{2} \mathrm{O}_{2}$ were divided into two aliquots and one of them was reduced with $N$-methylmercaptoacetamide (MMA), a reagent highly specific for the chemical reduction of methionine sulfoxide (23). Untreated, oxidized, and MMA-treated samples were then precipitated with methanol, washed extensively, analyzed by SDS-PAGE in the absence of $\beta$-mercaptoethanol, and then immunoblotted. Figure $3 \mathrm{C}$ shows the results of the oxidationreduction experiments for the $\operatorname{rSHaPrP}(23-232)$ chain, folded to both the $\alpha$ - and $\beta$-folds. Oxidation of the protein forms did not affect their recognition by IPC1, but the oxidative treatment caused a size increase, which was reversed following the reduction with MMA. Interestingly, oxidation of $\alpha$-rSHaPrP(23-232) was more efficient than that of $\beta$-rSHaPrP $(23-232)$, at least as judged by the extension of the size enhancement (apparent at $3 \mathrm{mM} \mathrm{H}_{2} \mathrm{O}_{2}$ for the $\alpha$-form but requiring $50 \mathrm{mM}$ in the case of the $\beta$-form). Immunoblotting the same samples with IPC2 shows a $\mathrm{H}_{2} \mathrm{O}_{2}$ concentration-dependent decrease in the degree of recognition of the PrP chains, which parallels the increase in the molecular size detected by IPC1. Again, such a decrease in the PrP immunoreactivity was more efficient for the $\alpha$-form than for the $\beta$-form and, in both cases, was reversed by MMA. Surprisingly, and consistent with previous results (17), the recognition of $\operatorname{rSHaPrP}(23-232)$ with $\alpha \operatorname{PrP}$ mAb 3F4, whose epitope comprises two methionines, M109 and M112, was not affected by oxidation (not shown).

Methionine Residues in $\operatorname{PrP}^{S c}$ Are Strongly Oxidized. Given the relevance of methionine oxidation, in particular of M213, with respect to the recognition of distinct PrP forms 

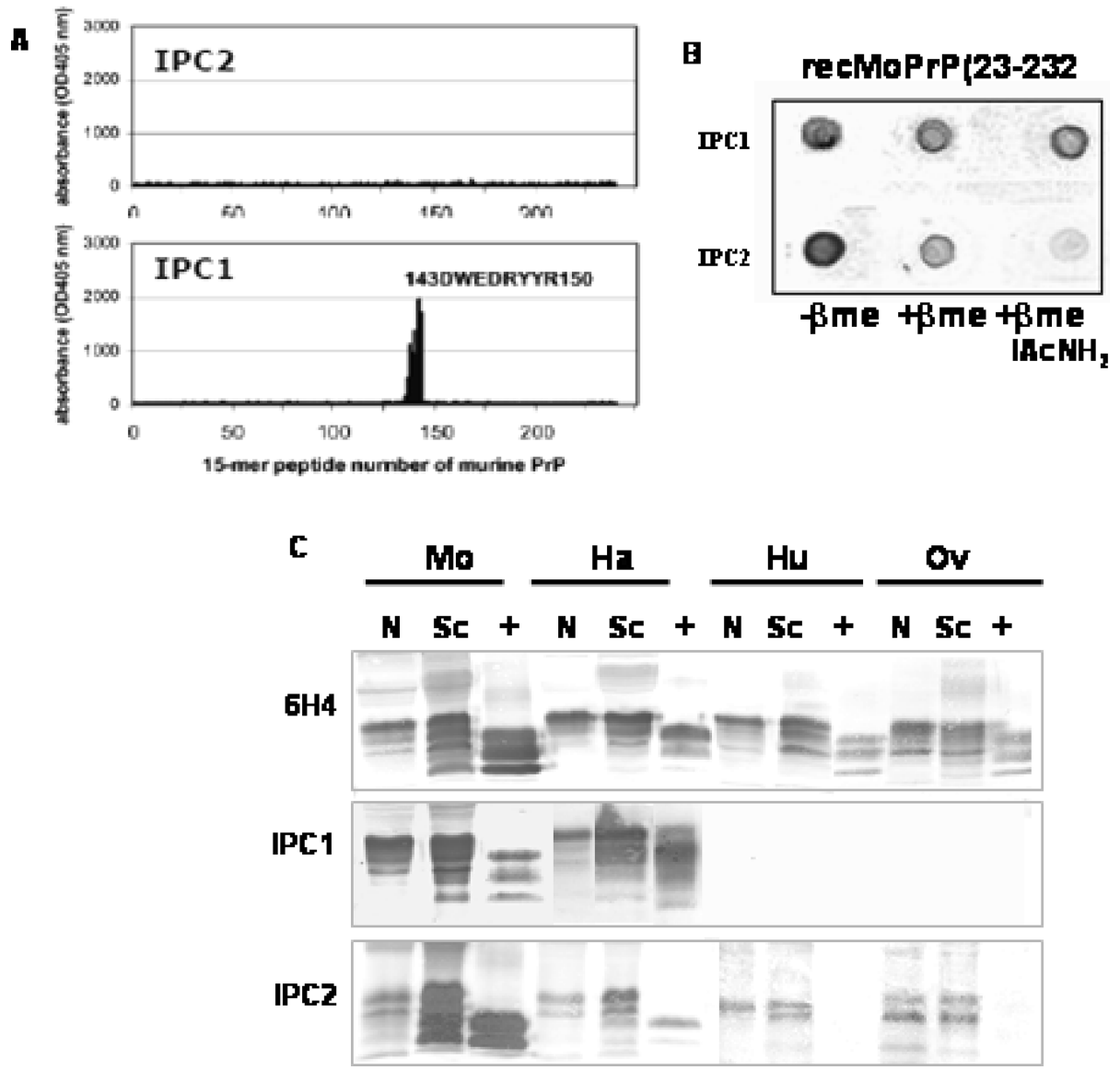

FIGURE 2: IPC2 epitope that comprises an oxidized disulfide bond. (A) PEPscan analysis of IPC1 (bottom) and IPC2 (top) anti-PrP mAbs. (B) Effect of reversible $(+\beta \mathrm{me})$ and irreversible $\left(+\beta\right.$ me $\left.\mathrm{IAcNH}_{2}\right)$ reduction of disulfide bonds on the recognition of recMoPrP $(23-230)$ by IPC1 and IPC2 analyzed by dot blot. Blot loads correspond to $10 \mathrm{ng}$ of protein. The analysis displayed in panels A-C was carried out in duplicate, varying both the sample source and the batch. (C) Typical immunoblots of normal (N) and prion-infected brain homogenates before $(\mathrm{Sc})$ or after proteinase $\mathrm{K}$ digestion $(+)$ of mouse $(\mathrm{Mo})$, hamster $(\mathrm{Ha})$, human $(\mathrm{Hu})$, and ovine (Ov) samples with 6H4, IPC1, and IPC2 anti-PrP mAbs. Lanes correspond to the load of $10 \mu \mathrm{L}$ of the original $10 \%(\mathrm{w} / \mathrm{v})$ homogenate. All samples were prepared in the absence of reducing reagents.

by IPC2, we then analyzed the oxidation state of the different methionine residues in PrP forms by MS/MS using the trypsin-digested Coomassie blue-stained gel bands (Figure $4 \mathrm{~A})$. The tested samples included enriched $\operatorname{SHaPrP}(27-30)$, untreated $\alpha$-rSHaPrP(23-232), and $\alpha$ - and $\beta$-rSHaPrP(23-232) forms oxidized with $50 \mathrm{mM} \mathrm{H}_{2} \mathrm{O}_{2}$. Of the different PrP tryptic peptides, we focused on those corresponding to residues 111-136 and 199-220.

Figure 4B describes the results obtained for the 199-220 tryptic peptide (VVEQMCTTQYQK), which is part of helix $\mathrm{C}$ of the PrP structure and contains M213 (29). We found that while this residue was not oxidized in the untreated recPrP, it becomes totally replaced by methionine sulfoxide (MetO) in the peptides obtained from $\mathrm{H}_{2} \mathrm{O}_{2}$-treated $\mathrm{PrP}$ chains. These results reinforced the conclusions of the immunoblotting experiments depicted in Figure 3C, in which IPC2 did not react with oxidized recPrP due to the oxidation of M213. They also indicate that Met/MetO oxidation is indeed an intrinsic feature of the sample under analysis and not a side reaction due to the biochemical manipulations required for MS/MS studies. The analysis of the band corresponding to the fully glycosylated $\operatorname{PrP}(27-30)$ revealed an oxidation pattern similar to that previously described by Stahl et al. (9), indicating the presence of MetO213 in a considerable fraction of $\mathrm{HaPrP}^{\mathrm{Sc}}$ molecules as an intrinsic feature.
Figure 4C shows the MS/MS results corresponding to the region of residues 111-136 (HMAGAAAAGAVVGGLGGYMLGSAMSR), which contains M112, M129, and M134. This peptide is part of the amyloidogenic region of PrP and also contains residue M129, which is an important polymorphic site modulating the susceptibility to disease acquisition and the disorder phenotype in humans (30). As depicted in the figure, while this region appears largely unaltered in untreated recPrP [only a negligible fraction of molecules exhibit all Met as MetO, probably resulting from the $\mathrm{Cu}(\mathrm{II})-$ catalyzed oxidative folding (28)], $\mathrm{H}_{2} \mathrm{O}_{2}$ treatment of the $\alpha$ and $\beta$-forms causes the quantitative formation of $\mathrm{MetO}(\mathrm{M} / \mathrm{Z}$ 804 representing MetOx3). At this point, the harshness of the oxidation protocol used impedes the assessment of whether this region exhibits a different predisposition to oxidation in the distinct folds. In contrast to untreated recPrP, the peptides from $\mathrm{HaPrP}^{\mathrm{Sc}}$ lack any nonoxidized Met (Figure $3 \mathrm{C})$. However, opposed to the oxidized recPrP samples, only a subpopulation of chains of $\operatorname{PrP}(27-30)$ were oxidized at all Met residues. Interestingly, MetO129 appears only when all the other Met residues of this peptide are oxidized. This suggests that $\mathrm{PrP}^{\mathrm{Sc}}$ aggregates are a heterogeneous mixture of chains exhibiting a complex pattern of Met/MetO combinations.

Differential Oxidation of Methionines in $\operatorname{PrP}^{C}$ and $\operatorname{Pr} P^{S c}$. The observed oxidation pattern implies that $\operatorname{PrP}^{\mathrm{C}}$ may also 
A
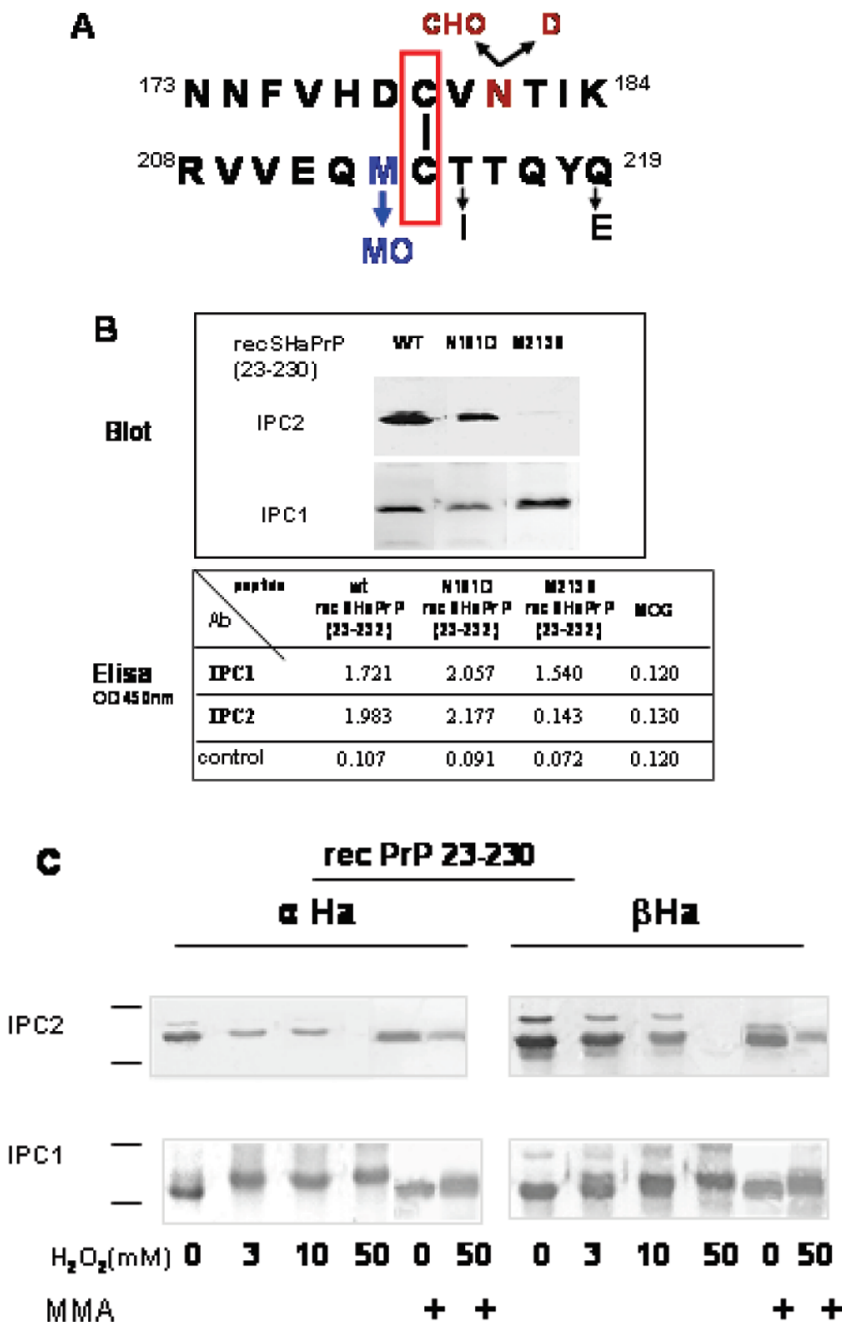

FIGURE 3: Oxidation of PrP M213 hinders IPC2 recognition. (A) SHaPrP sequence of the segments of helices B and C in the proximity of the disulfide bond. (B) IPC2 specificity probed by a Western blot and an ELISA using wild-type rSHaPrP(23-232) and its N181D and M213S mutants. (C) Effect of the oxidation with $\mathrm{H}_{2} \mathrm{O}_{2}$ and/or reduction with MMA of $\operatorname{rSHaPrP}(23-232)$ chains folded to their $\alpha-$ and $\beta$-forms on its recognition by IPC1 and IPC2 anti-PrP mAbs. Samples were denatured in the absence of $\beta$ me before their SDS-PAGE analysis.

be a good substrate for oxidation. However, due to the complex biochemical manipulations required for its purification, the direct analysis by mass spectrometry to establish the oxidation status of the diverse Met residues in native $\mathrm{PrP}^{\mathrm{C}}$ is far from trivial $(31,32)$. Nevertheless, we can gain insight into the oxidation status of M213 by utilizing the peculiar reactivity properties of IPC2. This can be done by testing whether chemical reduction of MetO, alone or in combination with in situ PNGase F-catalyzed deglycosylation, would allow the recognition of the previously undetected PrP chains by IPC2. For this purpose, normal (for $\mathrm{PrP}^{\mathrm{C}}$ ) and proteinase $\mathrm{K}$-digested prion-infected (for $\mathrm{PrP}^{\mathrm{Sc}}$ ) brain homogenates were incubated in the absence and presence of MMA, then resolved by SDS-PAGE in the absence of $\beta$-mercaptoethanol, and transferred to nitrocellulose membranes. In contrast to the irreversibility of the MetO reduction by MMA, Cys residues undergo reversible oxidation as required for recognition by IPC2. Subsequently, nitrocellulose membranes comprising the MMA-treated and untreated samples were incubated in the presence or absence of PNGaseF, to test the relative change in the recognition of individual PrP bands.

Figure 5A shows the results of these experiments for $\operatorname{PrP}^{\mathrm{Sc}}$. In both $\mathrm{MoPrP}^{\mathrm{Sc}}$ and $\mathrm{HaPrP}{ }^{\mathrm{Sc}}$, reduction with MMA rendered the fully glycosylated PrP chains partially recognizable by IPC2. These results are consistent with the mass spectrometry analysis indicating the oxidation of M213 in a large fraction of $\mathrm{HaPrP}^{\mathrm{Sc}}$ chains. Interestingly, in situ deglycosylation also favors the recognition of these PrP forms by IPC2. As depicted in the figure, digestion with PNGase $\mathrm{F}$ in the absence of MMA treatment had no effect on the recognition of $\mathrm{PrP}^{\mathrm{Sc}}$ bands by IPC2 in both $\mathrm{MoPrP}^{\mathrm{Sc}}$ and $\mathrm{HaPrP}^{\mathrm{Sc}}$, indicating that oxidation of M213 is a definite obstacle for the recognition of $\mathrm{PrP}^{\mathrm{Sc}}$ bands by this antibody. The most interesting results were obtained for the $\mathrm{HuPrP}^{\mathrm{Sc}}$ samples. While the untreated prion form of the human PrP was totally undetected by IPC2, MMA reduction primarily revealed the band corresponding to monoglycosylated $\mathrm{PrP}^{\mathrm{Sc}}$. It was only after an additional PNGase F digestion that the diglycosylated chain of $\mathrm{HuPrP}^{\mathrm{Sc}}$ was revealed by the IPC2 antibody. Similar results were obtained for OvPrPsc samples (not shown). These results suggest that in species other than rodents, M213 is also present as MetO in the non- and monoglycosylated $\mathrm{PrP}^{\mathrm{Sc}}$ chains.

Opposite results were obtained for samples containing $\operatorname{PrP}^{\mathrm{C}}$ (Figure 5B). In this case, reduction with MMA did not change the recognition pattern of $\operatorname{PrP}^{\mathrm{C}}$ by IPC2 while 


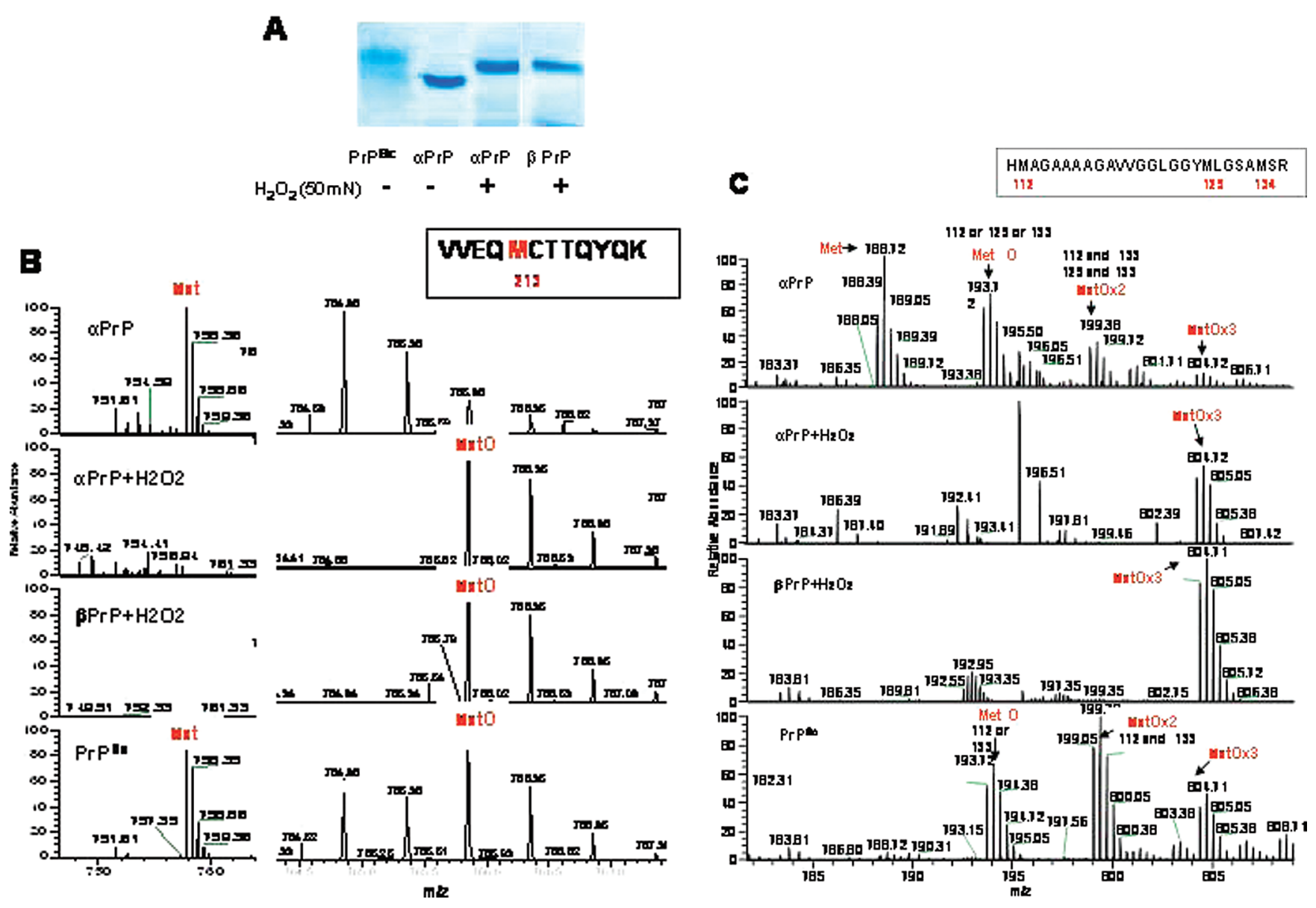

Figure 4: Proteinase K resistant $\mathrm{SHaPrPSc}$ and rSHaPrP(23-232) differ in their oxidation state of methionine residues. (A) Coomassie blue bands excised for trypsin digestion and mass spectrometry analysis. Typical mass spectrometry (MS/MS) spectra of the SHaPrP tryptic peptides corresponding to residues (B) 209-213 and (C) 111-136. Analysis was performed in duplicate using at least two independent samples.
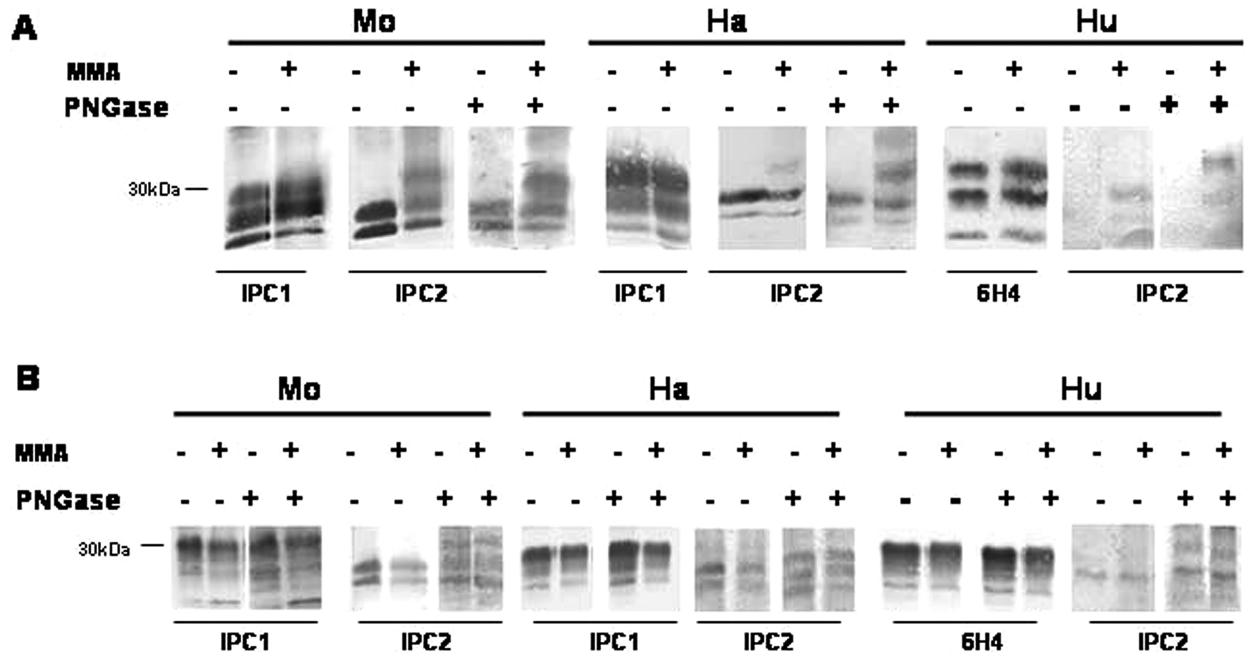

FIgURE 5: Differences in (A) proteinase $\mathrm{K}$ resistant $\mathrm{PrPSc}^{\mathrm{Sc}}$ and (B) $\mathrm{PrP}^{\mathrm{C}}$ by IPC2 are modulated by methionine oxidation and glycosylation. Mouse (Mo), hamster $(\mathrm{Ha})$, and human $(\mathrm{Hu})$ brain homogenates were incubated in the absence and presence of MMA, before electrophoretic analysis in the absence of $\beta$ me. After electrotransfer, nitrocellulose membranes were either directly blocked or in situ-digested with PNGase $\mathrm{F}$ before being immunoblotted with IPC1 (mouse and hamster) or 6H4 (human) anti-PrP mAbs. Displayed data are the typical results for three independent analyses. Lane loads correspond to $10 \mu \mathrm{L}$ of brain homogenates.

deglycosylation was sufficiently strong to allow the identification by IPC 2 of the chain corresponding to the highest-molecular weight band of $\operatorname{PrP}^{\mathrm{C}}$ in several species. These results indicate that as opposed to the case in $\mathrm{PrP}^{\mathrm{Sc}}$, M213 is not oxidized in $\operatorname{PrP}^{\mathrm{C}}$.

\section{DISCUSSION}

We have shown here that while methionine residues are oxidized in $\beta$-fold $\operatorname{PrP}^{\mathrm{Sc}}$, this is not the case for its $\alpha$-helical form, $\mathrm{PrP}^{\mathrm{C}}$. This finding indicates that the prion isoforms 
differ not only in their conformation but also in their covalent structure, constituting the first evidence of a prion-specific covalent signature. Our results pave the way for the production of prion-specific reagents in the form of antibodies against oxidized PrP peptides. They also suggest new strategies for the search of prion-related prophylactic therapies. Most importantly, the interaction between ROS and the metabolism of the prion proteins mechanistically relates prion-induced neurodegeneration with the pathogenesis of other conditions affected by the oxidative state of cells, such as amyotrophic lateral sclerosis, as well as Alzheimer's and Parkinson's diseases $(33,34)$.

From a structural point of view, while conversion of $\operatorname{PrP}^{\mathrm{C}}$ into $\mathrm{PrP}^{\mathrm{Sc}}$ has been uniquely ascribed to a conformational change, the finding of a covalent modification in the prion state of PrP allows new inducing or stabilizing factors to play a role in the complex process of prion formation. In this sense, since oxidized methionines (in particular M129 and M213) are contained in the chain regions identified as amyloid nucleating sites and protected from the solvent $(35,36)$, it seems reasonable to propose that oxidation, which mainly occurs on exposed residues, takes place when PrP is partially unfolded.

Our results also support the notion that the prion aggregate comprises a variety of PrP chains differing from each other in the number and position of oxidized methionines. This heterogeneity may correlate with diverse pathways of prion generation. For instance, oxidation can take place during the formation of the $\beta$-PrP molecules present in normal brain (37) or otherwise during $\operatorname{PrP}^{\mathrm{C}}$ misfolding.

Our results show that while $\alpha-\operatorname{recPrP}$ is readily oxidized by $\mathrm{H}_{2} \mathrm{O}_{2}$, this is not the case for the $\operatorname{PrP}^{\mathrm{C}}$ present in brains. This suggests a mechanism in which oxidized $\mathrm{PrP}^{\mathrm{C}}$ is efficiently reduced by the methionine sulfoxide reductase system, is rapidly degraded, or both. In contrast, the oxidized state of $\mathrm{PrP}^{\mathrm{Ps}}$ indicates that this form is a poor substrate for the methionine sulfoxide reductase systems or that the activity of these enzymes is impaired during disease, as in the case of aging (10). Such oxidized $\mathrm{PrP}^{\mathrm{Sc}}$ molecules may consequently promote their perpetuation by inhibition of the proteasome activity (38), therefore allowing the accumulation of additional aberrantly oxidized and folded PrP molecules $(39,40)$.

Lastly, whether the efficiency of the infectivity transmission and the properties of prion strains are related to a specific PrP methionine sulfoxide pattern remains to be established.

\section{REFERENCES}

1. Prusiner, S. B., Scott, M. R., DeArmond, S. J., and Cohen, F. E. (1998) Prion protein biology. Cell 93 (3), 337-348.

2. Pan, K. M., Baldwin, M., Nguyen, J., Gasset, M., Serban, A., Groth, D., Mehlhorn, I., Huang, Z., Fletterick, R. J., Cohen, F. E., et al. (1993) Conversion of $\alpha$-helices into $\beta$-sheets features in the formation of the scrapie prion proteins. Proc. Natl. Acad. Sci. U.S.A. 90 (23), 10962-10966.

3. Naslavsky, N., Stein, R., Yanai, A., Friedlander, G., and Taraboulos, A. (1997) Characterization of detergent-insoluble complexes containing the cellular prion protein and its scrapie isoform. J. Biol. Chem. 272 (10), 6324-6331.

4. Taraboulos, A., Rogers, M., Borchelt, D. R., McKinley, M. P., Scott, M., Serban, D., and Prusiner, S. B. (1990) Acquisition of protease resistance by prion proteins in scrapie-infected cells does not require asparagine-linked glycosylation. Proc. Natl. Acad. Sci. U.S.A. 87 (21), 8262-8266.
5. Chesebro, B., Trifilo, M., Race, R., Meade-White, K., Teng, C., LaCasse, R., Raymond, L., Favara, C., Baron, G., Priola, S., Caughey, B., Masliah, E., and Oldstone, M. (2005) Anchorless prion protein results in infectious amyloid disease without clinical scrapie. Science 308, 1435-1439.

6. Neuendorf, E., Weber, A., Saalmueller, A., Schatzl, H., Reifenberg, K., Pfaff, E., and Groschup, M. H. (2004) Glycosylation deficiency at either one of the two glycan attachment sites of cellular prion protein preserves susceptibility to bovine spongiform encephalopathy and scrapie infections. J. Biol. Chem. 279, 53306-53316.

7. Riek, R., Hornemann, S., Wider, G., Billeter, M., Glockshuber, R., and Wuthrich, K. (1996) NMR structure of the mouse prion protein domain PrP(121-321). Nature 382 (6587), 180-182.

8. Somerville, R. A., Millson, G. C., and Kimberlin, R. H. (1980) Sensitivity of scrapie infectivity to detergents and 2-mercaptoethanol. Intervirology 13, 126-129.

9. Stahl, N., Baldwin, M. A., Teplow, D. B., Hood, L., Gibson, B. W., Burlingame, A. L., and Prusiner, S. B. (1993) Structural studies of the scrapie prion protein using mass spectrometry and amino acid sequencing. Biochemistry 32 (8), 1991-2002.

10. Stadtman, E. R. (2001) Protein oxidation in aging and age-related diseases. Ann. N.Y. Acad. Sci. 928, 22-38.

11. Petropoulos, I., and Friguet, B. (2006) Maintenance of proteins and aging: The role of oxidized protein repair. Free Radical Res. 40, 1269-1276.

12. Glaser, C. B., Yamin, G., Uversky, V. N., and Fink, A. L. (2005) Methionine oxidation, $\alpha$-synuclein and Parkinson's disease. Biochim. Biophys. Acta 1703, 157-169.

13. Brock, J. W., Ames, J. M., Thorpe, S. R., and Baynes, J. W. (2007) Formation of methionine sulfoxide during glycoxidation and lipoxidation of ribonuclease A. Arch. Biochem. Biophys. 457, 170 176.

14. Brown, D. R. (2005) Neurodegeneration and oxidative stress: Prion disease results from loss of antioxidant defence. Folia Neuropathol. 43, 229-243.

15. Nadal, R. C., Abdelraheim, S. R., Brazier, M. W., Rigby, S. E., Brown, D. R., and Viles, J. H. (2007) Prion protein does not redoxsilence $\mathrm{Cu}^{2+}$, but is a sacrificial quencher of hydroxyl radicals. Free Radical Biol. Med. 42, 79-89.

16. Redecke, L., von Bergen, M., Clos, J., Konarev, P. V., Svergun, D. I., Fittschen, U. E., Broekaert, J. A., Bruns, O., Georgieva, D., Mandelkow, E., Genov, N., and Betzel, C. (2007) Structural characterization of $\beta$-sheeted oligomers formed on the pathway of oxidative prion protein aggregation in vitro. J. Struct. Biol. 157, 308-320.

17. Requena, J. R., Dimitrova, M. N., Legname, G., Teijeira, S., Prusiner, S. B., and Levine, R. L. (2004) Oxidation of methionine residues in the prion protein by hydrogen peroxide. Arch. Biochem. Biophys. 432, 188-195.

18. Breydo, L., Bocharova, O. V., Makarava, N., Salnikov, V. V., Anderson, M., and Baskakov, I. V. (2005) Methionine oxidation interferes with conversion of the prion protein into the fibrillar proteinase K-resistant conformation. Biochemistry 44, 15534 15543.

19. Gonzalez-Iglesias, R., Pajares, M. A., Ocal, C., Espinosa, J. C., Oesch, B., and Gasset, M. (2002) Prion protein interaction with glycosaminoglycan occurs with the formation of oligomeric complexes stabilized by $\mathrm{Cu}$ (II) bridges. J. Mol. Biol. 319, 527540.

20. Legname, G., Baskakov, I. V., Nguyen, H. O., Riesner, D., Cohen, F. E., DeArmond, S. J., and Prusiner, S. B. (2004) Synthetic mammalian prions. Science 305, 673-676.

21. Bueler, H., Fischer, M., Lang, Y., Bluethmann, H., Lipp, H. P., DeArmond, S. J., Prusiner, S. B., Aguet, M., and Weissmann, C. (1992) Normal development and behaviour of mice lacking the neuronal cell-surface PrP protein. Nature 356 (6370), 577-582.

22. Boshuizen, R. S., Langeveld, J. P., Salmona, M., Williams, A., Meloen, R. H., and Langedijk, J. P. (2004) An in vitro screening assay based on synthetic prion protein peptides for identification of fibril-interfering compounds. Anal. Biochem. 333, 372-380.

23. Houghten, R. A., and Li, C. H. (1979) Reduction of sulfoxides in peptides and proteins. Anal. Biochem. 98, 36-46.

24. Vretou, E., Giannikopoulou, P., and Psarrou, E. (2001) Polymorphic outer-membrane proteins of Chlamydophila abortus are glycosylated. Microbiology 147, 3303-3310.

25. Rosenfeld, J., Capdevielle, J., Guillemot, J. C., and Ferrara, P. (1992) In-gel digestion of proteins for internal sequence analysis after one- or two-dimensional gel electrophoresis. Anal. Biochem. 203, 173-179. 
26. Wilm, M., and Mann, M. (1996) Analytical properties of the nanoelectrospray ion source. Anal. Chem. 68, 1-8.

27. Riesner, D. (2003) Biochemistry and structure of $\operatorname{PrP}(C)$ and $\operatorname{PrP}(\mathrm{Sc})$. Br. Med. Bull. 66, 21-33.

28. Gonzalez-Iglesias, R., Elvira, G., Rodriguez-Navarro, J. A., Velez, M., Calero, M., Pajares, M. A., and Gasset, M. (2004) $\mathrm{Cu}^{2+}$ binding triggers $\alpha$ BoPrP assembly into insoluble laminar polymers. FEBS Lett. 556, 161-166.

29. Riek, R., Hornemann, S., Wider, G., Billeter, M., Glockshuber, R., and Wuthrich, K. (1996) NMR structure of the mouse prion protein domain $\operatorname{PrP}(121-321)$. Nature 382, 180-182.

30. Palmer, M. S., Dryden, A. J., Hughes, J. T., and Collinge, J. (1991) Homozygous prion protein genotype predisposes to sporadic Creutzfeldt-Jakob disease. Nature 352 (6333), 340-342. [Erratum, (1991) 352 (6335), 547].

31. Turk, E., Teplow, D. B., Hood, L. E., and Prusiner, S. B. (1988) Purification and properties of the cellular and scrapie hamster prion proteins. Eur. J. Biochem. 176 (1), 21-30.

32. Pan, K. M., Stahl, N., and Prusiner, S. B. (1992) Purification and properties of the cellular prion protein from Syrian hamster brain. Protein Sci. 1 (10), 1343-1352.

33. Varadarajan, S., Yatin, S., Aksenova, M., and Butterfield, D. A. (2000) Review: Alzheimer's amyloid $\beta$-peptide-associated free radical oxidative stress and neurotoxicity. J. Struct. Biol. 130, 184-208.

34. Chi, L., Ke, Y., Luo, C., Gozal, D., and Liu, R. (2007) Depletion of reduced glutathione enhances motor neuron degeneration in vitro and in vivo. Neuroscience 144, 991-1003.
35. Sun, Y., Breydo, L., Makarava, N., Yang, Q., Bocharova, O. V., 573 and Baskakov, I. V. (2007) Site-specific conformational studies 574 of prion protein (PrP) amyloid fibrils revealed two cooperative 575 folding domains within amyloid structure. J. Biol. Chem. 282, 576 9090-9097.

36. Cobb, N. J., Sonnichsen, F. D., McHaourab, H., and Surewicz, W. K. (2007) Molecular architecture of human prion protein amyloid: A parallel, in-register $\beta$-structure. Proc. Natl. Acad. Sci. U.S.A. 104, 18946-18951.

37. Yuan, J., Xia, X., McGeehan, J, Dong, Z, Cali, I, Fujioka, H., Kong, Q., Kneale, G., Gambetti, P., and Zou, W. Q. (2006) Insoluble aggregates and protease-resistant conformers of prion protein in uninfected human brains. J. Biol. Chem. 281, 34848 34858.

38. Kristiansen, M., Deriziotis, P., Dimcheff, D. E., Jackson, G. S., Ovaa, H., Naumann, H., Clarke, A. R., van Leeuwen, F. W., Menendez-Benito, V., Dantuma, N. P., Portis, J. L., Collinge, J., and Tabrizi, S. J. (2007) Disease-associated prion protein oligomers inhibit the 26S proteasome. Mol. Cell 26, 175-188.

39. Ciechanover, A., and Brundin, P. (2003) The ubiquitin proteasome system in neurodegenerative diseases: Sometimes the chicken, sometimes the egg. Neuron 40, 427-446.

40. Yedidia, Y., Horonchik, L., Tzaban, S., Yanai, A., and Taraboulos, A. (2001) Proteasomes and ubiquitin are involved in the turnover of the wild-type prion protein. EMBO J. 20, 5383-5391. 